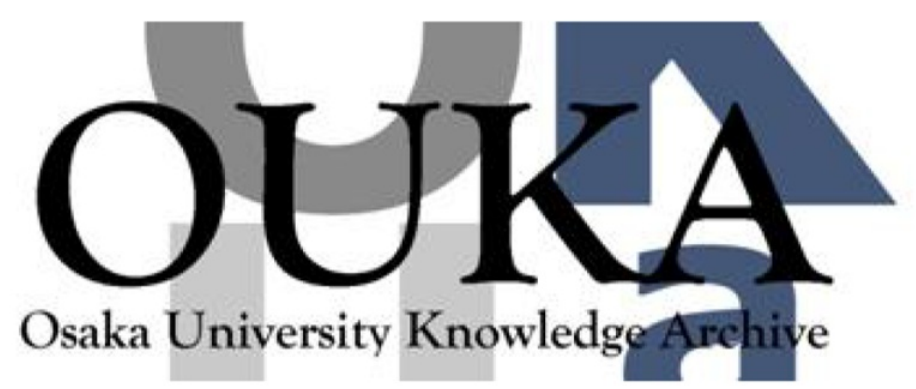

\begin{tabular}{|c|l|}
\hline Title & $\begin{array}{l}\text { Electrical and optical properties of discotic } \\
\text { liquid crystals with various core structures }\end{array}$ \\
\hline Author(s) & Azumai, R.; Ozaki, M. ; Yoshino, K. et al. \\
\hline Citation & $\begin{array}{l}\text { IEEE International Conference on Conduction and } \\
\text { Breakdown in Dielectric Liquids, ICDL. p. 397- } \\
\text { p.400 }\end{array}$ \\
\hline Issue Date & $2002-07$ \\
\hline oaire:version & VoR \\
\hline URL & $\begin{array}{l}\text { https://hdl. handle. net/11094/14055 } \\
\text { c2002 IEEE. Personal use of this material is } \\
\text { permitted. However, permission to } \\
\text { reprint/republish this material for advert is ing } \\
\text { or promotional purposes or for creat ing new } \\
\text { collective works for resale or redistribution } \\
\text { to servers or lists, or to reuse any } \\
\text { copyrighted component of this work in other } \\
\text { works must be obtained from the IEEE. }\end{array}$ \\
\hline rights \\
\hline Note & \begin{tabular}{l} 
\\
\hline
\end{tabular} \\
\hline
\end{tabular}

Osaka University Knowledge Archive : OUKA

https://ir. Library. osaka-u. ac. jp/

0saka University 


\title{
Electrical and Optical Properties of Discotic Liquid Crystals with Various Core Structures
}

\author{
R. Azumai, M.Ozaki, K.Yoshino and K.Ohta* \\ Department of Electronic Engineering, Faculty of Engineering, Osaka University \\ Yamada-Oka 2-1, Suita; Osaka 55-0871 Japan \\ *Department of Functional Polymer Science, Faculty of Textile Science and Technology, \\ Shinshu University, Ueda 386-8567 Japan
}

\begin{abstract}
Electrical and optical properties in discotic liquid crystals having various core structures such as triphenylene, phthalocyanine, metal phthalocyanine and other aromatic moieties have been studied. The electrical conductivity, photoconductivity and carrier mobility are measured as a function of temperature and molecular alignment. The anisotropy of the conductivity and carrier mobility have been confirmed. The molecular alignment characteristics of various discotic liquid crystals have also been investigated by means of a polarizing microscope observation. An excellent uniform molecular alignment, in which the columnar axis aligns perpendicular to the substrates, has been observed in the tetragonal columnar phase of liquid crystal with rare-earth phthalocyanine core.
\end{abstract}

\section{INTRODUCTION}

Contrary to organic liquids in which free electrons with large mobility depending on the molecular structure are confirmed to exist $[1,2]$, only ionic carrier mobility, which is related to the viscosity of the fluid by Walden's rule, has been reported in ordinary liquid crystals such as nematic, smectic and cholesteric liquid crystals $[3,4]$. However, recently, observations of hole mobility have been reported in discotic liquid crystals such as 2,3,6,7,10,11-Hexahexyloxytriphenylene (HHOTP) in which disc-shaped aromatic units stack in one direction and form a columnar structure[5-9], although direct evidence of hole transport is not complete. This is very important finding for the understanding of carrier transport process in fluids, because the report on direct observation of holes in dielectric liquids is highly limited so far.

Since the discovery of the hole mobility in HHOTP, many studies on the electrical conduction have been carried out. In most studies, however, discotic liquid crystal with triphenylene core or similar structures of the core have been used for the experiments. In this paper, we report the electrical and optical properties and molecular alignment characteristics in discotic liquid crystals with various core such as triphenylene, phthalocyanine, metal phthalocyanine and other aromatic moieties.

\section{EXPERIMENTAL}

The molecular structures of discotic liquid crystals used in this study are shown in Fig.1. HHOTP was synthesized and purified by a column chromatography utilizing silica gel and chloroform/

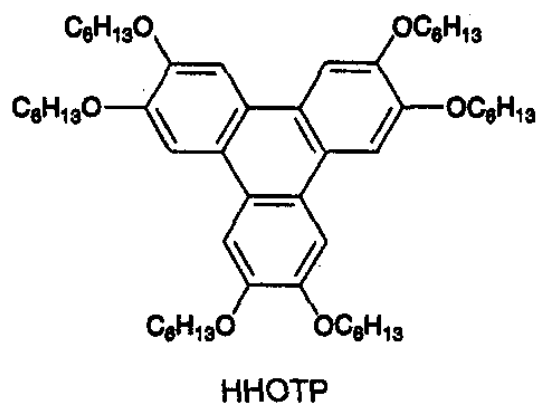

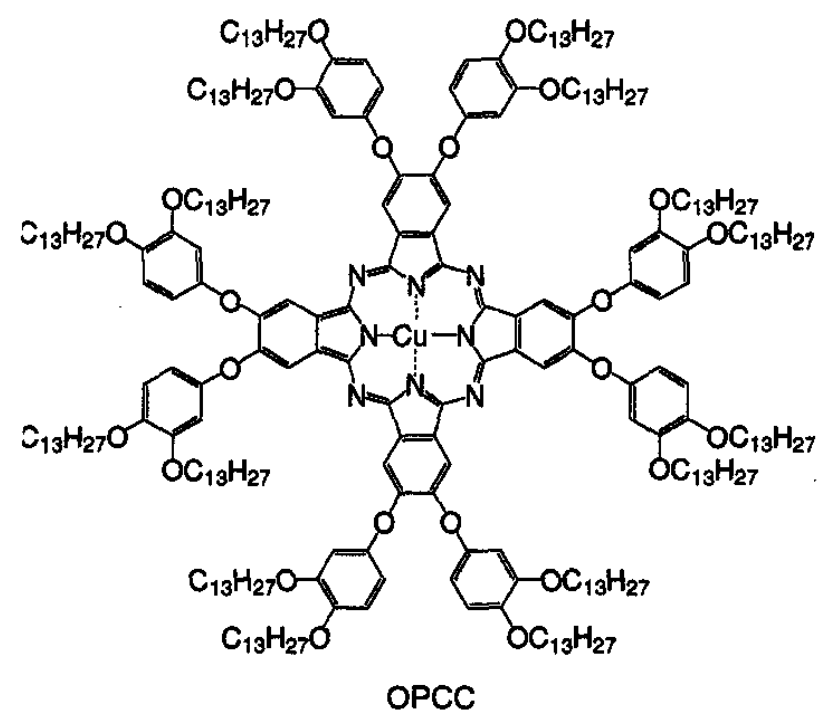

Fig. 1. Molecular structures of discotic liquid crystals used in this study. 


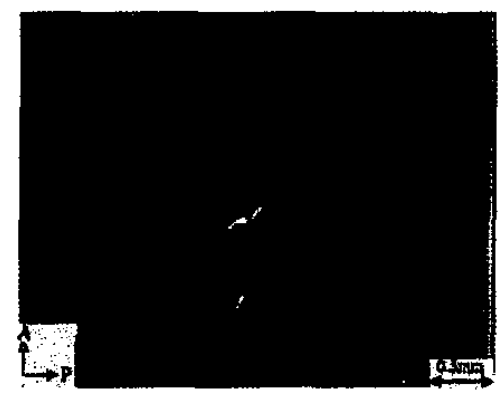

Fig. 2. Polarizing microphotograph of HHOTP in the discotic phase.

benzene mixture (4/1) as eluent, and then recrystallized from acetone [10]. In optical microscopic observation, the discotic phase of HHOTP was confirmed in the temperature range between $53^{\circ} \mathrm{C}$ and $96^{\circ} \mathrm{C}$. The detailed procedure of the synthesis of OPCC has previously been reported in [11]. The phase transition temperature of OPCC was also confirmed by the polarizing microscope observation.

The sample was introduced in a sandwich cell, which was composed of two parallel quartz plates with various separations depending on the thickness of the polyethylene-terephthalate (PET) sheets used as spacers, by capillary action in the isotropic phase. In this study, two types of electrode configurations were used for the measurement of anisotropy of electrical conduction.
One has two ITO (In-Sn oxide) electrodes on both substrates (sandwich cell). Another one has an inter-digit electrode on one of substrates (inter-digit cell). The conductivity and mobility parallel and perpendicular to the column were measured using sandwich and inter-digit cells, respectively. By cooling the temperature of the cell slowly from the isotropic phase, molecular alignment with a columnar structure oriented perpendicularly to substrates was realized in the discotic phase, which was confirmed by optical microscope observation. In the sandwich cell, the electric field is applied parallel to the columnar axis, while in the inter-digit cell, the field is perpendicular to the column.

The carrier mobility was evaluated by a time-of-flight method [12] utilizing the third harmonic generation (THG) of Q-switched $\mathrm{Nd}$ :YAG light $(355 \mathrm{~nm})$ with $20 \mathrm{~ns}$ in pulse width as an exciting light source. The photon flux was measured utilizing a portable power meter (Gentec SERIES3130). The conductivity was measured with a digital electrometer (Keithley 617).

\section{RESULTS AND DISCUSSION}

Figure 2 shows a polarizing microphotograph of the columnar phase of HHOTP. A homeotropic alignment of the molecule is confirmed. In general, the director of the discotic liquid crystal is defined as the direction normal to the core plane of the molecules. In a homeotropically aligned cell of the discotic liquid
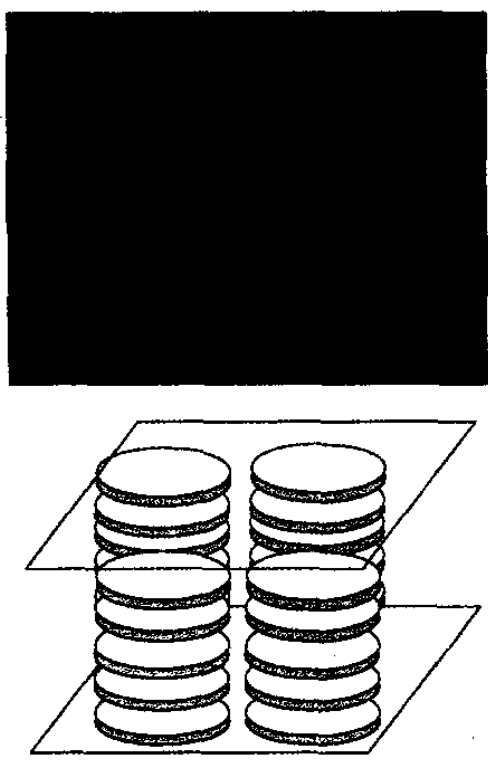

(a)
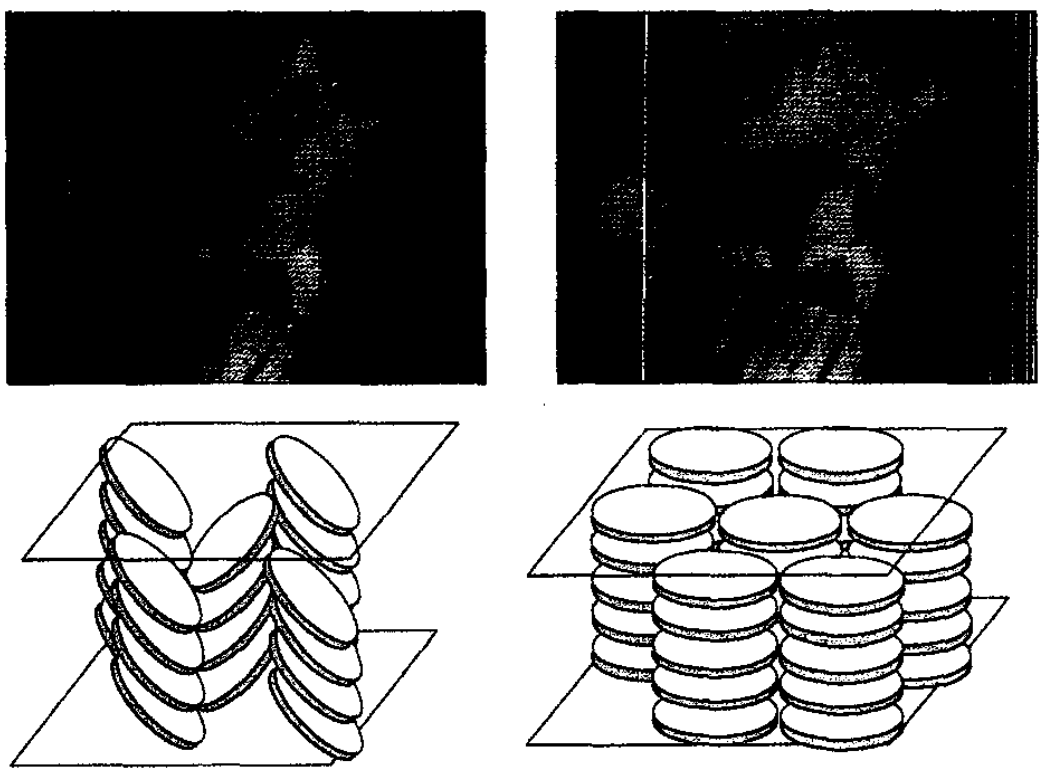

(b)

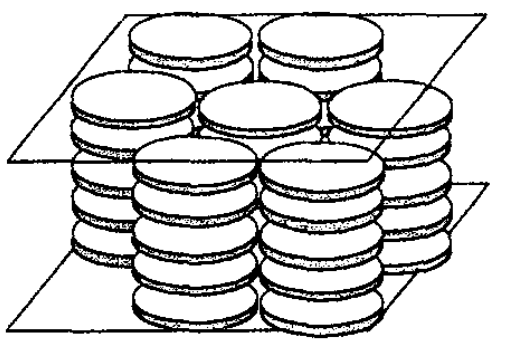

(c)

Fig. 3. Polarizing microphotographs and schematic explanation of the molecular alignment of OPCC in tetragonal (a), rectangular (b) and hexagonal (c) columnar phases. 
crystal, the director is perpendicular to the substrates. Therefore, if the molecules are homeotropically aligned in the cell, there is no optical anisotropy in the cell plane because of the symmetric molecular shape and alignment, resulting in the extinction in the polarizing microscope observation as shown in Fig.2. As is evident from Fig.2, however, domain structures are observed and a complete uniform alignment was not obtained.

Figure 3 shows a polarizing microphotograph in the tetragonal, rectangular and hexagonal columnar phases of OPCC. The schematic explanations are also shown in Fig.3. In the hexagonal and rectangular phases, domain structures are observed in the same manner as HHOTP. It should be noted, however, that perfect extinction is observed in the tetragonal phase as shown in Fig.3(a). This indicates that a complete homeotropic alignment is obtained. So far, a homeotropic alignment has never been observed in the columnar phase of the discotic liquid crystal having phthalocyanine core in the molecule. It has also been confirmed that the homeotropic alignment in the rectangular phase of OPCC has excellent structural stability. The alignment was not influenced by a mechanical shear stress, thermal shock, surface roughness of the substrates and electrohydrodynamic flow.

The electrical properties such as electrical conductivity and carrier mobility have been investigated. Figure 4 shows the temperature dependence of electrical conductivity in HHOTP. In this case, the measurement was carried out during a cooling stage with a cooling rate of $0.2^{\circ} \mathrm{C} / \mathrm{min}$. It is clearly shown in this figure that the conductivity in the direction parallel to the column is much higher than that for the perpendicular direction in the columnar phase. Moreover, the difference of an activation energy in the discotic phase indicates that the conduction mechanism along the columnar structure is different from that across the columnar axis.

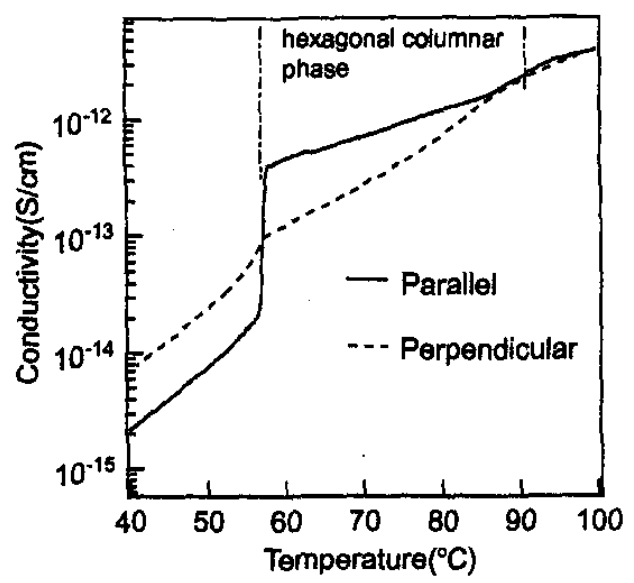

Fig. 4. Temperature dependence of electrical conductivity parallel and perpendicular to the columnar axis of HHOTP.

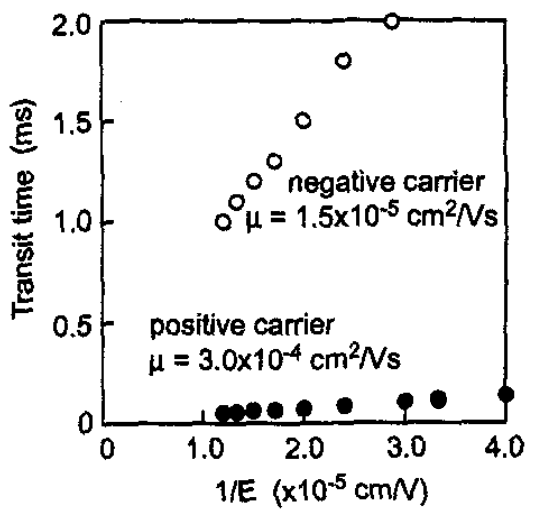

Fig. 5. Field dependence of transit time for positive and negative cartiers in HHOTP evaluated from a time-of-flight measurement.

Figure 5 shows the field dependence of the transit time for both positive and negative carriers in HHOTP. From this figure, it is found that the mobility for the positive carrier was much larger than that for the negative one.

In order to evaluate the mobility for the carrier migration perpendicular to the columnar axis, the inter-digit cell was used for the time-of-flight measurement. Figure 6 shows the cell structure of the inter-digit electrode. When the carrier density $n_{0}$ emerged by exciting light pulse is constant between electrodes and the carrier are traveling by the applied voltage at the velocity $v_{d}$, total current density $J(t)$, which drifts between electrodes at time $t$, is given as the integration of the charge carrier density [13]:

$$
J(t)=\frac{e_{0} n_{0} v_{d}}{d} \int_{v_{d}}^{d} d x=J_{0}\left(1-\frac{t}{t_{d}}\right)
$$

where $d$ is the distance between electrodes, $J_{0}$ is the initial current density at $t=0$ and $t_{\mathrm{d}}$ is the transit time of the carrier.

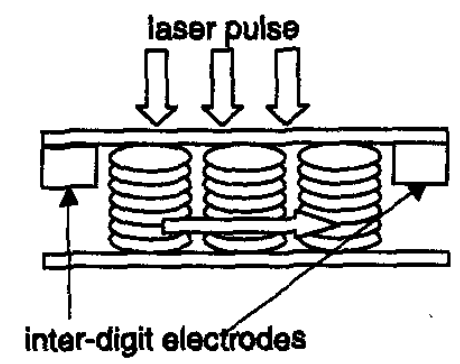

Fig. 6. Cell structure of the inter-digit electrode for the measurement of carrier mobility perpendicular to the columnar axis. 


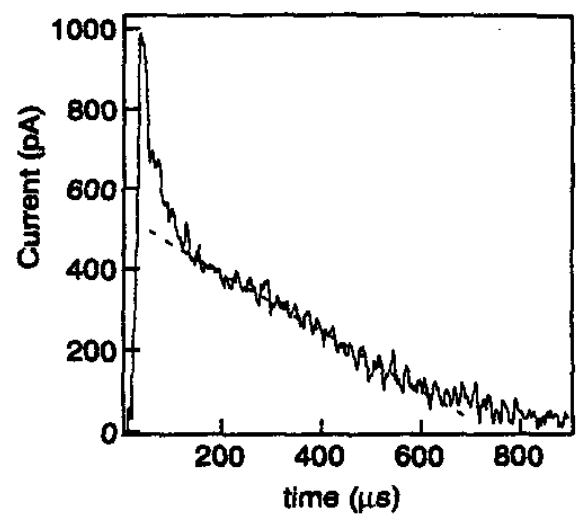

Fig. 7. Typical waveform of the transient photocurrent in the inter-digit cell of HHOTP.

Figure 7 shows an experimental waveform of transient photocurrent in the inter-digit cell. From this transit time, we can estimate the mobility in the direction perpendicular to the columnar axis to be $1.8 \times 10^{-4} \mathrm{~cm}^{2} / \mathrm{Vs}$, which is smaller than that in the direction parallel to the columnar axis, as shown in Fig.8.

\section{SUMMARY}

Electrical and optical properties in discotic liquid crystals with various core structures were investigated. The high hole mobility in the columnar phase of HHOTP was confirmed by means of time-of flight measurement. The anisotropy of the electrical conductivity and carrier mobility were observed. An excellent uniform homeotropic alignment was observed in the tetragonal columnar phase of OPCC and the structural stability of this alignment was also confirmed.

\section{REFERENCES}

[1] Schmidt W. F. and Allen A. O., J. Chem. Phys., Vol.50, 1969, pp.5037.

[2] Minday R. M., Schmidt L. O. and Davis H. T., J. Chem. Phys., Vol.50, 1969, pp.1473.

[3] Yoshino K., Hisamitsu S. and Inuishi Y., "Temperature dependence of mobility in p-azoxyanisole", J. Phys. Soc. Jpn., Vol.32, No.3, March, 1972, pp.867.

[4] Yoshino K., Tanaka N. and Inuishi Y., "Anomalous Carrier Mobility in Smectic Liquid Crystal", Jpn. J. Appl. Phys., Vol.15, No.4, 1976, pp.735-736.

[5] Adam D., Rokhildt W. and Haarer D., "Charge-Carrier Transport in Liquid-Crystal Based Organic Photoconductors",

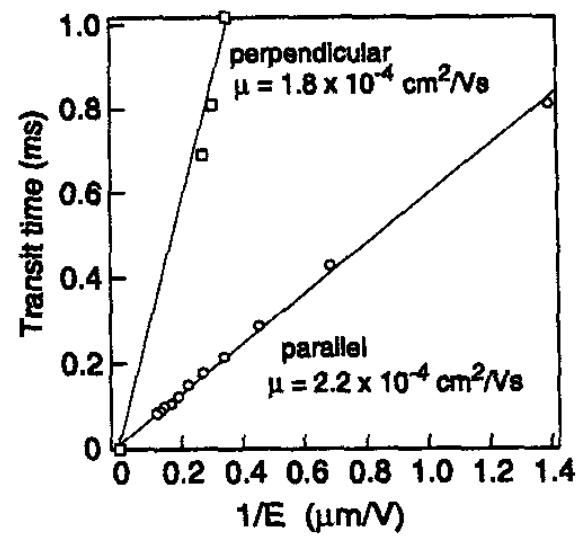

Fig. 8. Field dependence of the transit time of positive carriers in the direction parallel and perpendicular to the columnar axis of HHOTP.

Jpn. J. Appl. Phys., Vol.35, Part 1, No. 3, 15 March, 1996, pp.1826-1831.

[6] Adam D., Closs F., Frey T., Funhoff D., Haarer D., Rigsdorf H., Schuhmacher P. and Siemensmeyer K., "Transient photoconductivity in a discotic liquid crystal", Phys. Rev. Lett. Vol.70, No.4, 25 January, 1993, pp.457-460.

[7] Yoshino K., Nakayama H., Ozaki M., Onoda M. and Hamaguchi M., "Electrical Properties of Discotic Liquid Crystal Hexahexyloxytriphenylene", Jpn. J. Appl. Phys., Vo.36, Part 1, No. 8, 15 August, 1997, pp.5183-5186.

[8] Nakayama H., Ozaki M., Schmidt W.F. and Yoshino K., "Measurements of Carrier Mobility and Quantum Yield of Carrier Generation in Discotic Liquid Crystal HexahexylOxytriphenylene by Time-of-Flight Method", Jpn. J. Appl. Phys., Vo.38, Part 2, No. 9A/B, 15 September, 1999, pp.L1038-L1041.

[9] Ohta K., Ban K., Nishizawa K., Warman J.M., Craats A.M., Ozaki M. and Yoshino K., Discotic Liquid Crystals as New Class of Fluid and Their Electrical and Optical Properties", Proc. 13th Int. Conf. Dielectric Liq. (ICDL'99), 99CH36213, pp.561-566.

[10] Chiang L. Y., Safinya C. R., Clark N. A., Liang K. S. and Bloch A. N., J. Chem. Soc. Chem. Commun., 1985, pp.695.

[11] Hatsusaka K., Ohta K., Yamamoto I. and Shirai H., "Discotic Liquid Crystals of Transition Metal Complexes, Part 30.: Spontaneous Uniform Homeotropic Alignment of Octakis (dialkoxyphenyl) phthalocyaninatocopper (II) Complexes", J. Mater. Chem., Vol.11, 2001, pp.423-433.

[12] Yoshino K., "Carrier Mobility Measurement", IEEE Trans. Electr. Insul., Vo.21, No.6, December, 1986, pp.999-1006.

[13] Schmidt W.F., "Liquid State Electronics of Insulating Liquids", CRC Press, New York, 1997, pp.58. 